\title{
POÉTICA: DESCRIPCIÓN Y EVALUACIÓN
}

\author{
MILTON H. SNOEYENBOS \\ Georgia State University
}

Una teoría de la poesía, una poética, abarca como mínimo los tópicos de identificación, descripción y evaluación. En este artículo asumo que podemos identificar poemas y diferenciarlos de cualquier otra cosa. Asumo también que se puede establecer una lista de las propiedades descriptivas que posee un poema. Supuesto que podemos identificar y describir poemas, consideraré el tema de la evaluación en el contexto del siguiente diálogo entre un esteta $(\mathrm{T})$ y un escéptico $(\mathrm{S})$.

(TI): El poema $P_{1}$ es mejor que el poema $P_{2}$.

(S2) : ¿Por qué?

(T3) : $\stackrel{P}{1}_{1}$ posee, y $P_{2}$ carece de $E_{1}$... $E_{n}$ (donde las Ees son propiedades tales como claridad de imágenes, unidad, originalidad, etcétera).

(S4) : Pero, ¿cómo puede usted decir que $P_{1}$ posee, y que $P_{2}$ carece de $E_{1} \ldots E_{n}$ ? ¿No es esto precisamente una cuestión de gusto?

Más aún, si (T) puede dar una respuesta a (S4), el escéptico todavia puede recalcar más la proposición en estos términos:

(S5): Dado que $P_{1}$ posee, y $P_{2}$ carece de $E_{1} \ldots E_{n}$, ¿por qué $E_{1} \ldots E_{n}$ hacen a $P_{1}$ mejor que $P_{2}$ ? ¿No es esto precisamente una cuestión de gústo?

El propósito de este trabajo es proporcionar una estrategia general para refutar las posiciones (S4) y (S5). 
Para empezar, pues, asumo que podemos identificar poemas. Pienso que se puede dar una explicación general de nuestra habilidad para identificar poemas y géneros poéticos, pero, habiendo discutido esto en otro lugar," no lo tocaré en esta ocasión. Asumo también que se puede obtener un conjunto indefinido de descripciones verdaderas de cualquier poema. Este conjunto es indefinido porque de cualquier poema que contenga $k$ palabras puede decirse con verdad que contiene menos palabras que $k+n$, donde $n$ es cualquier entero mayor que $\mathrm{O}$. Pero del conjunto de descripciones verdaderas solamente un subgrupo serán descripciones apropiadas. En tanto la pregunta sobre cuáles son los rasgos o características apropiados de un poema sigue siendo difícil, sugiero que una respuesta adecuada deberá tener en cuenta los intereses y necesidades que, de hecho, la poesía satisface. Aunque puede ser verdad que un poema contenga menos palabras que $k+n$, tal cosa no parece satisfacer en forma alguna cualquiera de nuestros intereses, ni tener ninguna relación con propiedades de poemas que satisfacen nuestros intereses. Así, esto no es apropiado para la descripción y evaluación de un poema. Pienso que la necesidad de una explicación psicológicamente fundamentada de las descripciones apropiadas, y la importancia de tal explicación para nuestro tópico de evaluación, se hacen evidentes si consideramos la siguiente clase de situación.?

Supóngase que usted sostiene en las manos dos pequeños objetos ovalados y que solo se le dice que provienen originariamente de la estrella Alfa, de la constelación Centauro. $0_{1}$ resplandece débilmente y está algo caliente, mientras que $0_{2}$ brilla espléndidamente y está frío. Ambos emiten un sonido parecido al de una viola. Usted puede, por supuesto, describir parcialmente ambos objetos. Pero supóngase ahora

1 Snoeyenbos, Milton, "Theory and Genre Concepts", Philosophical Forum, 8 (1976), pp. 87-93; "Art.: Theory and Definition"', Southern Journal of Philiosophy, 15 (1977), pp. 227-38; "Vagueness and Art", British Journal of Aesthetics, 18 (1978), pp. 12-18.

2 Debo este tipo de ejemplo al Profesor Keith Gunderson de la Universidad de Minnesota. 
que le preguntan a usted si $0_{1}$ es bueno, o si $0_{2}$ es mejor que $0_{1}$. Aunque se le diga que las propiedades que usted notó son apropiadas para la cuestión evaluativa, usted estaría perplejo al responder, puesto que hasta donde usted sabe, los objetos pueden ser astronaves o formas de vida. Suponga ahora que los objetos son identificados como obras de arte, y que usted descubre que el sonido es la lengua de Centauro. Supóngase que $0_{1}$ elabora con lujo de detalles la verdadera leyenda de la fundación de la sociedad centaura, mientras que $\mathrm{O}_{2}$, al contar la leyenda, omite algunos detalles importantes, falsificando el resto. Supóngase además que los habitantes de Centauro tienen un gran interés en que su leyenda sea contada con toda exactitud y con lujo de detalles. Finalmente, suponga que el calor y el débil resplandor de $0_{1}$ facilitan la atención centaura, añadiendo a la crónica cierta unidad, equilibrio y gracia, mientras que el brillo frío de $\mathrm{O}_{2}$ distrae a los centauros introduciendo un elemento de distorsión e incoherencia.

El punto está en que, dada la información concerniente a los intereses y necesidades de Centauro, puede proveerse una lista de descripciones apropiadas. A su vez, las descripciones apropiadas, más una explicación de los intereses y necesidades, proporcionan una base para la evaluación. $\theta_{1}$ es mejor que $0_{2}$ porque posee ciertas notas descriptivas de las que $0_{2}$ carece, y si la aplicabilidad para la evaluación de un aspecto descriptivo se somete a prueba, ello está justificado en términos de los. intereses y necesidades que los habitantes de Centauro de hecho poseen. Yo sugiero que la situación es análoga en el caso de la poesía, y que nuestro pequeño cuento proporciona la base para una respuesta adecuada a (S4) y (S5).

Los críticos ciertamente evalúan los poemas y aducen razones para sus evaluaciones, donde una razón es una declaración sobre alguna: propiedad descriptiva del poema en cuestión. Puesto que estamos familiarizados con los poemas en una manera en la que no lo estamos con las obras de arte 
de Centauro, de la práctica crítica en sí puede obtenerse un inventario de descripciones apropiadas para la evaluación. Éste incluirá: imágenes claras, individualidad y consistencia de la delineación del carácter, originalidad, coherencia y unidad de los varios aspectos estructurales del poema, frescura y propiedad de los modos linguísticos, como la metáfora y cosas así. Si un críticu defiende que $P_{1}$ es mejor que $P_{2}$, porque $P_{1}$ es más consistente, tiene imágenes más claras y es más original, está citando razones descriptivas para su evaluación. Una razón crítica es, por lo tanto, una propiedad (o relación, como en el caso de la originalidad) que contribuye a que un poema sea bueno o malo; añade o quita algo al mérito del poema. Cuando, como en (S5), se pone en duda la aplicabilidad de una razón crítica, ésta puede justificarse, por lo menos en parte, sobre la base de los intereses y necesidades que la gente de hecho posee. Suponga usted, por ejemplo, que la gente tiene un interés en comunicar descripciones y una necesidad de variedad en la percepción sensorial. En este caso, nuestro diálogo crítico puede desarrollarse como sigue:

(T1): El poema $\mathrm{P}_{1}$ es mejor que el $\mathrm{P}_{2}$.

(S2) : ¿Por qué?

(T3): En gran medida porque el conjunto de imágenes de $\mathrm{P}_{1}$ es más claro que el de $\mathrm{P}_{2}$, y $\mathrm{P}_{1}$ es más original.

$\mathrm{Si}$ asumimos que se puede dar una respuesta a (S4), de manera que $P_{1}$ es en efecto más claro en imágenes y más original, entonces nuestro problema está en:

(S5) : ¿Por qué las imágenes claras y la originalidad hacen a $\mathrm{P}_{1}$ mejor que $\mathrm{P}_{2}$ ? ¿No es esto una cuestión de gusto?

Dado que la gente tiene los intereses y necesidades que hemos supuesto, nuestra respuesta a (S5) será algo como esto: 
(T6) : Porque la gente está interesada en comunicar descripciones, y la claridad de las imágenes permite satisfacer este interés. Más aún, la originalidad de $P_{1}$ satisface nuestra necesidad de una variedad de percepciones sensoriales, mientras que la repetición en $\mathrm{P}_{2}$ conduce al fastidio y no satisface nuestra necesidad de variedad en las percepciones sensoriales.

Ahora bien, una respuesta como la de (T6) a (S5) presupone que (S4) ha sido contestada, porque el escéptico insiste en que el hecho de que un poema sea consistente, unificado, original, etcétera, es una cuestión de gusto, o está "en el ojo del espectador". La versión contemporánea del escepticismo es la afirmación de que términos estéticos tales como "unificado" y "coherente" no están regidos por propiedades que son suficientes para su correcta aplicación. Examinemos los argumentos de Frank Sibley para esta conclusión. ${ }^{3}$ Desde un principio argüiré que él falla al presentar su caso y que hay propiedades suficientes para el uso de tales términos. Si este es el caso, (S4) no tiene sustentación.

Sibley dice que hay una distinción de sentido común entre dos subclases de la clase de predicados descriptivos - estéticos (E) y no estéticos (N). Predicados típicos N son: "es un soneto", "tiene 14 líneas", "tiene 108 palabras" y "está en pentámetro yámbico". Predicados típicos E son: "está integrado", "tiene imágenes claras", "es consistente" y "está equilibrado". Aunque los predicados N y E son ambos descriptivos, Sibley dice que los términos $\mathbf{N}$ representan propiedades de objetos que pueden ser reconocidas por cualquiera con ojos, oídos e inteligencia normales, mientras que los términos E se refieren a propiedades cuyo reconocimiento requiere el ejercicio del gusto o de la sensibilidad. Esta diferencia en las propiedades está reflejada lingüísticamente

:3 Sibley, Frank, "Aesthetic Concepts". Philosophical Review, 68 (1959), pp. 421-50; "Aesthetic and Nonaesthetic", Philosophical Review, 74 (1965), pp. 135.59. 
en que, mientras algunos términos $\mathrm{N}$, tales como "cuadrado", se aplican sobre la base de propiedades necesarias y suficientes, y otros términos $\mathrm{N}$, tales como "inteligente", se aplican sobre la base de propiedades suficientes, el hecho de no hallarse regidos de esta manera es una de las características clave de los términos E. Sibley ofrece tres argumentos para su conclusión de que no hay propiedades suficientes para el uso adecuado de un término $\mathrm{E}$.

Sibley concede que ciertas propiedades $\mathrm{N}$ pueden verse típicamente asociadas con una propiedad $\mathrm{E}, \mathrm{E}_{1}$. De este modo podemos decir que cuando un objeto tiene estas propiedades $N$, tiene también con frecuencia propiedades $E_{1}$. Pero él dice que las propiedades $\mathrm{N}$ pueden asociarce también con una propiedad $\mathrm{E}$ completamente distinta, $\mathrm{E}_{2}$. Por ejemplo, la propiedad de la gracia o elegancia visual se asocia frecuentemente con propiedades $\mathrm{N}$, tales como la ligereza, agilidad y suavidad de curvas. Pero estas propiedades $\mathrm{N}$ no bastan para que una pintura sea elegante, porque con frecuencia se asocian también con la propiedad E de insipidez.

Este es un argumento débil. Podemos encontrar que algunas propiedades $\mathrm{N}$ asociadas con la gracia o la elegancia están asociadas con la insipidez o la insulsez, pero no todas. Las propiedades que capacitan para derrotar a los ajedrecistas promedio, para realizar cómputos aritméticos y para seguir simples deducciones lógicas, están típicamente asociadas tanto con personas inteligentes como con computadoras convenientemente programadas; sin embargo, no se sigue de aquí que todo el conjunto de propiedades asociadas con personas inteligentes sea idéntico al conjunto de propiedades asociadas con una computadora. Para que el argumento de Sibley fuera persuasivo, tendría que presentarnos un análisis detallado de propiedades $\mathrm{N}$ que estén típicamente asociadas con el uso de términos tales como "gracioso" $\mathrm{e}$ "insípido". Pero él no ofrece tal análisis.

Sibley desarrolla su segundo argumento en el curso de una discusión de términos $\mathrm{N}$. Concede que especificar las 
propiedades suficientes para un término $\mathrm{N}$ como "inteligente" puede ser una tarea difícil, pero dice que podemos escoger ejemplos que poseen tales propiedades. Del estudio de tales ejemplos podemos derivar principios que nos permitan aplicar correctamente los términos $\mathrm{N}$ a nuevos casos.

Ahora bien, este argumento no diferencia los términos $\mathrm{N}$ de los términos E. Suponga que usted sabe que yo poseo un IQ de 170, que derroto regularmente a los expertos del ajedrez, y que puedo resolver complejos enigmas lógicos. Todavía es posible que usted, conociendo estos hechos, se pregunte si yo soy realmente inteligente. En tal caso nos sentiríamos inclinados a decir que usted simplemente desconoce las propiedades para el uso propio de "inteligente", y que esta falta de conocimiento constituye la base para su actitud de duda. De modo análogo, su vacilación sobre si un poema tiene realmente un conjunto de imágenes claras puede fundarse en su desconocimiento de las propiedades suficientes para el uso de "imágenes claras". Así, hay un paralelo entre términos $\mathrm{N}$ y $\mathrm{E}$, no una diferencia. Más aún, Sibley sostiene que nosotros no podemos establecer principios para términos $\mathrm{E}$, pero que los podemos establecer para términos N. Puesto que esto es precisamente lo que él tiene que probar, su argumento es una petición de principio. En consecuencia, el segundo argumento de Sibley no justifica una distinción entre términos $\mathrm{N}$ y $\mathrm{E}$.

En su tercer argumento, Sibley establece que si los términos $\mathrm{E}$ están regidos por propiedades suficientes, podríamos formular un conjunto de reglas para cada término y una yersona podría aplicar los términos en forma mecánica, con completa confianza en que su aplicación sería correcta. Sin embargo, él sostiene que un novicio o principiante en estética, a quien se dé un conjunto de reglas para términos $\mathrm{E}$, no las podría aplicar con confianza. Por lo tanto, no hay propiedades suficientes para el uso de un término $\mathrm{E}$.

Ahora bien, parecería que no hay diferencia a este res. pecto entre términos $\mathrm{N}$ y $\mathrm{E}$. Suponga usted que yo no sé 
cómo usar el término $\mathrm{E}$ "equilibrado" $\mathrm{y}$ el término $\mathrm{N}$ "inteligente", y que entonces usted me proporciona las reglas " $\mathrm{X}$ es inteligente si $X$ es $N_{1} \ldots N_{1}, "$ y "X es equilibrado si. $X$ es $\mathrm{N}_{k}$.... $\mathrm{N}_{n}$." Cuando yo empiece a aplicar las reglas puedo muy bien hacerlo con vacilación. Después de todo, solamente he recibido un conjunto de reglas, y el mismo Sibley reconoce, con respecto al término $\mathrm{N}$ "inteligente", que las reglas pueden ser complejas y que puede ser difícil o imposible dar reglas que digan cuántas propiedades se necesitan para un conjunto suficiente. Por lo tanto parecería que cualquier vacilación que yo mostrara se aplicaría igualmente al uso de ambos términos $\mathrm{N}$ y $\mathrm{E}$. Pero supóngase que a mí se me ha dado el conjunto de reglas. Entonces no hay razón para pensar que yo no tendría confianza de haber aplicado las reglas con éxito. Por lo tanto, en el argumento:

Premisa $_{1}$ : Si hubiera suficientes propiedades para la aplicación de términos $\mathrm{E}$, entonces los términos $\mathrm{E}$ podrian aplicarse con confianza por parte de cualquiera a quien se proporcionara la lista de las propiedades;

Premisa $_{2}$ : Pero los términos E no podrían aplicarse con confianza por parte de cualquiera a quien se proporcionara la lista de las propiedadies;

Conclusión: Luego, no hay propiedades suficientes para la aplicación de términos $\mathrm{E}$,

la segunda premisa es falsa si asumimos que a la persona se le ha proporcionado el conjunto correcto de reglas. Más aún, no parecería haber distinción aquí entre los términos $\mathrm{N}$ y E. Supongamos que Sibley, a pesar de nuestro ejem. plo en contra, insistiera en que la segunda premisa es verdadera. Su insistencia tendría que estar basada en la asunción de que no hay propiedades suficientes para el uso co. rrecto de términos E. Pero esta es la conclusión que hay que establecer. El resultado final es que stu argumento tiene una premisa falsa o es circular. 
Sibley falla al establecer que no hay propiedades suficientes para el uso de un término $E$ y consecuentemente falla al establecer una distancia o vacío lógico entre los términos $\mathrm{N}$ y E. Además, supóngase que no hay propiedades necesarias ni suficientes para el uso correcto de un término E. En. tonces para cualquier conjunto de propiedades $N_{1} \ldots N_{n}$, el caso no es que un objeto $\mathrm{O}$ sea $\mathrm{E}$ si y solamente si $\mathrm{O}$ tiene $N_{1} \ldots N_{n}$, ni que $O$ sea $E$ solamente si $O$ tiene $N_{1} \ldots N_{n}$, ni que $O$ sea $E$ si $O$ tiene $N_{1} \ldots N_{n}$. El problema está en que en tal caso " $\mathrm{E}$ " se aplicaría correctamente a cualquier objeto. Si no hay propiedades sobre las que se base el uso del término, nunca será incorrecto aplicar el término a cualquier objeto. En consecuencia, si concedemos que términos $E$ tales como "claro conjunto de imágenes" se aplican correctamente a algunos poemas, pero no a todos los poemas, quiere decir que debe haber algunas propiedades en las que se base tal uso.

Por supuesto que tales propiedades han de concebirse de una manera no demasiado simplificada. Aunque tal vez no haya una propiedad $\mathrm{N}$ suficiente para el uso correcto de " $\mathrm{E}$ ", $O$ puede ser E si $\left(\left(N_{1}\right.\right.$ y $N_{2}$ y $\left.N_{3}\right)$ o $\left(N_{2}\right.$ y $N_{3}$ y $\left.N_{4}\right)$ o $\left(N_{3}\right.$ y $N_{4}$ y $\left.N_{5}\right)$ ). Tal clase puede también ser necesaria y suficiente, esto es, cada disyunción es suficiente y uno de los conjuntos de propiedades disyuntivas debe estar presente para que "E" se aplique. Por lo tanto, en general, deberíamos esperar encontrar alguna clase de propiedades suficien. tes por lo menos para el uso de un término $\mathrm{E}$, y en algunos caso tal vez podamos ser capaces de especificar una clase compleja de propiedades necesarias y suficientes. Si esto su. cede, la estrategia escéptica representada por (S4) puede ser refutada. A:

(S4) : ¿Cómo puede usted decir que $\mathrm{P}_{1}$ posee, y $\mathrm{P}_{2}$ carece de $E_{1} \ldots E_{n}$ ? ¿No es esto precisamente una cuestión de gusto?

la respuesta es: un poema posee $E_{1}$ si y solamente si posee 
$N_{1} \ldots: N_{n} ; P_{1}$ posee $N_{1} \ldots N_{n}$, pero $P_{2}$ no. Análogamente para $\mathbf{E}_{2} \ldots \mathbf{E}_{\mathrm{n}}$

Esto nos deja con la estrategia escéptica representada por (S5):

(S5): Dado que $P_{1}$ posee y $P_{2}$ carece de $E_{1} \ldots E_{n}$, ¿por qué $E_{1} \ldots E_{n}$ hacen a $P_{1}$ mejor que $P_{2}$ ? ¿No se trata justamente de una cuestión de gusto?

Como se ha indicado previamente en nuestra discusión sobre el ejemplo de Alfa Centauro, si la gente posee intereses y necesidades $I_{1} \ldots I_{n}$, entonces nuestra respuesta a (S5) es:

(T6): Porque la gente tiene $I_{1} \ldots I_{n} ;$ y la posesión de $E_{1} \ldots E_{n}$ por $P_{1}$ satisface $I_{1} \ldots I_{n}$, mientras que $P_{2}$, que carece de $E_{1} \ldots E_{n}$, no puede satisfacer $I_{1} \ldots I_{\text {. }}$.

Lo que yo sugiero, pues, es que la evaluación de la poesía es un tipo de evaluación funcional. La ventaja de este enfoque del problema es que las evaluaciones son fáciles de entender epistemológicamente. Considere el ejemplo de los cuchillos. Los humanos tenemos una variedad de intereses y necesidades, como por ejemplo, obtener alimento, que se satisfacen cortando y rebanando una variedad de objetos, por ejemplo, la carne. Los cuchillos realizan esta función; un cuchillo es un instrumento que consta de una hoja delgada que se usa para cortar. Si uno conoce la función de los cu. chillos, está en posición de investigar empíricamente qué propiedades de los cuchillos se requieren para que éstos posean una alta capacidad para el desarrollo de su función. Puesto que los cuchillos se consideran buenos o malos de acuerdo a cómo cortan, uno examina los cuchillos que cumplen con su función y los que no la realizan, y entonces extrae las propiedades poseídas por los cuchillos que tienen un alto nivel de capacidad cortante. En este caso tenemos propiedades tales como la dureza y la agudeza. Estas propiedades descriptivas sé convierten èn criterios de excelencia 
para los cuchillos. Cuando uno está consciente de estos criterios, uno está en posición de determinar objetivamente si un cuchillo en particular es bueno.

Los cuchillos tienen una función general, la de cortar; pero pueden aducirse más funciones específicas. Así, podemos hablar de cuchillos para cortar papel y de cuchillos para cortar carne. Frecuentemente, tal indicador preposicional no se mencionará en el discurso, y la función deberá ser especificada por el contexto. Funciones específicas pueden requerir criterios específicos. Mientras que " $O$ es un buen cuchillo" puede incluir criterios tales como dureza y agudeza, " $O$ es un buen cuchillo para cortar papel" puede incluir criterios tales como dureza, agudeza y pesadez. No hay, por tanto, nada extraño en decir de un cuchillo que es a la vez bueno y no bueno, queriendo indicar que es bueno en cierto aspecto (por ejemplo, para cortar carne, en cuyo caso la delgadez es un criterio), y no bueno en otro aspecto (digamos, para cortar papel, donde los criterios son el espesor y la pesadez).

En el caso de la poesía, empezamos con ciertos intereses y necesidades genéricas, tales como el interés o la necesidad de expandir nuestros horizontes cognoscitivos, imaginativos y emocionales. ${ }^{*}$ Otros intereses y necesidades, como el interés en comunicar información y la necesidad de una variedad de percepciones sensoriales, pueden situarse bajo intereses genéricos. Los poemas son un medio de satisfacer estos intereses. Así como los cuchillos nos permiten ejercer la actividad de cortar de tal manera que nuestro interés en asegurar el alimento pueda ser satisfecho, así los poemas nos capacitan para entregarnos a la actividad de leer de un modo tal que nuestro interés por dilatar nuestros horizontes cognoscitivos, imaginativos y emocionales pueda ser satisfecho. Si éstos son nuestros intereses en lo que hace a los poemas, estamos en posición de investigar empíricamente la cuestión

* Sigo aquí a Haig Khatchadourian en The Concept of Art (New York: New York University Press, 1971), pp: 47.70. 
de qué cualidades o características otorgan a los poemas un alto grado de capacidad para satisfacer estos intereses. Si, para tomar un ejemplo simplificado, como parte de nuestro interés general en extender nuestros horizontes cognoscitivos, tenemos interés en comunicar una descripción, entonces cualidades como la claridad, la coherencia, la profundidad y la sublimidad deben considerarse como propiedades poseídas por los poemas que tienen un alto nivel de capacidad para satisfacer este interés. Estos serán criterios de excelencia en poesía. Supuesto que podemos especificar los intereses y necesidades que la poesía debe satisfacer en primer lugar, el establecimiento de estas propiedades-criterio es una cuestión empírica.

Consideremos ahora algunos factores que complican nuestra explicación de la evaluación poética. En primer lugar, no deberíamos esperar encontrar en los poemas una sola propiedad que sea, a la vez, necesaria y suficiente para la excelencia poética. Pero de esto no se sigue que no haya conjuntos complejos de propiedades que sean suficientes para la excelencia poética. Considérese el caso de los cuchillos: quizás no todo cuchillo bueno para cortar carne sea delgado, y no todo cuchillo delgado sea bueno para cortar carne, pero la delgadez puede ser un criterio de los cuchillos buenos para cortar carne, si otras cosas permanecen iguales. $Y$ en combinación con otras propiedades tales como la dureza, la agudeza, etcétera, ciertamente parecería que tenemos un conjunto de propiedades suficientes para que un cuchillo sea bueno para cortar carne. Análogamente, aunque una deter. minada propiedad puede estar presente en algunos poemas pobres y por lo tanto ser insuficiente por sí sola, y estar ausente de algunos poemas buenos y por lo tanto ser innecesaria, puede sin embargo contribuir a la excelencia poética de cualquier poema que la contenga; puede ser una cualidad meritoria dondequiera que se encuentre. No todo buen poema puede ser coherente, $y$ no todo poema coherente puede ser bueno, pero la coherencia puede ser una característica de 
los buenos poemas, si otras cosas permanecen iguales. De este modo, parece razonable pensar que podríamos especificar conjuntos o series complejas de propiedades $\mathrm{E}$ que serían suficientes para la excelencia poética. Esquemáticamente: 0 es un buen poema si $\left(\left(E_{1} \ldots E_{k}\right)\right.$ o $\left(E_{2} \ldots E_{\jmath}\right) o \ldots$ $\left.\left(E_{\mathrm{j}} \ldots \mathrm{E}_{\mathrm{n}}\right)\right)$. Aquí no hay propiedades comunes entre la primera y la última disyunción; por lo tanto, ninguna propiedad es necesaria, pero cada una de las disyunciones es suficiente.

El hecho de que hay que considerar un número de propiedades introduce la posibilidad de que alguna de las propiedades que hacen que los poemas sean buenos no sea simultáneamente satisfactoria al máximo. Considérese los mapas de carreteras, cuya función es permitirle a uno llegar a un punto de destino. Puede ser que la minuciosidad en los detalles sea una propiedad de los buenos mapas de carreteras, de tal manera que cuanto más completo esté el mapa, mejor sea; pero la legibilidad es también una cualidad importante de los buenos mapas, y un mapa completo en todo detalle puede ser ilegible. Monroe Beardsley ha señalado que en algunos casos dos propiedades pueden ser de alguna manera incompatibles." Así como el valor y la sensibilidad son ambas propiedades meritorias que raramente se encuentran en un solo ser humano, así el humor y la tensión dramática raramente se encuentran combinados con éxito en la tragedia.

Beardsley señala un camino para afrontar este problema concediendo que algunas propiedades pueden subordinarse a otras. Si consideramos el género tragedia, la tensión dra. mática puede ser un criterio primario para la excelencia trágica, y cualquier otro criterio de menor rango, o secundario, puede ser meritorio en tanto acrecienta la tensión dramática, pero puede ser un demérito en cuanto disminuye dicha tensión. El humor, por ejemplo, puede ser un criterio secundario en el caso de la tragedia. El humor puede enriquecer la tragedia en la medida en que proporciona un alivio

D Beardsley, Monroe, "On the Generality of Critical Reasons", Journal of Philosophy, 59 (1962), pp. 447-86. 
momentáneo y, de ese modo, realza la tensión dramática. Pero si, por otra parte, el humor desvirtúa la tensión dramática, entonces es un demérito.

Una explicación razonable para una jerarquía de criterios podría establecerse si hay una jerarquía de intereses en la poesía. Esta es una cuestión para una investigación empírica, pero tal jerarquía es ciertamente plausible. Hemos supuesto anteriormente que un interés en expandir nuestros horizontes cognoscitivos, imaginativos y emocionales puede ser el interés primario que la poesía satisface, mientras que un interés en comunicar información y la necesidad de variedad de percepciones sensoriales pueden ser intereses secundarios. Es digno de notarse que Aristóteles ofreció básicamente este tipo de relación. Si estaba en lo correcto al decir que el interés o necesidad primario que debe satisfacer la tragedia es el placer concomitante a la purgación de las emociones por medio del temor y la piedad, entonces la tensión dramática bien puede considerarse un criterio más central que el humor, que proporciona solamente el placer de un alivio momentáneo.

La mención de los géneros introduce otro factor complicado que debe ser considerado. Hemos de distinguir entre las clases generales de comparación y las clases específicas y subespecíficas. La poesía es una clase general, el soneto es una clase específica, y los sonetos de Petrarca son una clase subespecífica. Los estetas, en gran parte, han estado preocupados con la clase muy general de comparación de las obras de arte. Sin embargo, puesto que los fines para los cuales se emplea un mayor número de clases específicas están típicamente más definidos que aquellos para los que se emplean clases generales, parecería que las propiedades de las clases específicas serían más fáciles de establecer. Piénsese, por ejemplo, en los muchos fines para los que se usan los cuchillos y en los intereses más específicos satisfechos por los cuchillos de carnicero. Las propiedades suficientes para la excelencia de la clase más específica serían 
ciertamente más fáciles de especificar. Esto sugiere que el punto de mira, o foco, del esteta sobre el arte debe estar balanceado por un punto de mira igual sobre los géneros específicos. Esto complica el trabajo de escoger las propiedades que subrayan o constituyen la bondad o excelencia de los objetos, pero no implica que el trabajo no pueda ser realizado.

Resumiendo: he esbozado una explicación de evaluación funcional para refutar el escepticismo representado por (S4) y (S5). He demostrado que Sibley falla al establecer (S4) y que no hay razones para pensar que los términos $\mathrm{E}$ no tienen propiedades suficientes para su correcta aplicación. Concediendo que existen ciertos intereses y necesidades satisfechos por la poesía -y la determinación de si los hay es una cuestión empírica- estamos entonces en posición de investigar empíricamente qué propiedades de los poemas les otorgan un alto nivel de capacidad para satisfacer estos intereses y necesidades. A lo largo de estas líneas, (T6) es nuestra respuesta a (S5). Finalmente, hemos considerado un número de factores que complican, pero no descartan ni eliminan, la explicación funcional de la evaluación poética; de hecho, el tratamiento o enfoque funcional con la explicación, cuidadosamente llevada a cabo, de una jerarquía de intereses $y$ necesidades puede muy bien proporcionar una base para comprender la admitidamente rica complejidad de la evaluación poética. 
A theory of poetry, a poetics, comprises at least three sets of themes: those about identification, description, and evaluation. In this paper it is assumed we are able to identify and describe poems. In order to narrow down on the remaining theme, that of evaluation, we are invited to consider the following dialogue between an esthete (T) and a skeptic (S).

(T1) : Poem $P_{1}$ is better than poem $P_{2}$.

(S2) : Why?

(T3) : $P_{1}$ has $E_{1} \ldots E_{n} ; P_{2}$ lacks those qualities. (Such as clearness of image, unity, originality, and so on.)

(S4) : But how can you say $P_{1}$ has, and $P_{2}$ lacks, $E_{1} \ldots E_{n}$ ? Isn't that just a matter of taste?

A stronger version of (S4) goes as follows:

(S5) : Given $P_{1}$ has, and $P_{2}$ lacks, $E_{1} \ldots, E_{n}$, why should $E_{1} \ldots$ $E_{n}$ make $P_{1}$ better than $P_{2}$ ? Isn't that just a matter of taste?

The purpose here is to draw a general strategy for refuting both (S4) and (S5).

Since we are able to identify poems, and also to obtain any number of true descriptions of any poem, a basis for evaluation is given by the appropiate descriptions, together with an explanation of the community's interests and needs.

When evaluating a poem, critics advance reasons, and a reason is a declaration about some quality descriptive of the poem in question. Appropiate descriptions for evaluation are, for instance, clear images, originality, the cohesive unity among the diversity of structural aspects, the freshness and propriety of the linguistic modes, such as metaphor, etc. A critical reason is, then, a quality which contributes to the poem being good or bad; it adds to, or detracts from, the poem's merit.

If, as in (S5), the aplicability of a critical reason is put in doubt, it can be justified on the basis of interests and needs that people in fact have. For instance, if people have an interest on communicating descriptions, and a need for variety in sensorial perception, clear 
images and originality in a poem work for the fulfillment of those interests and needs. In such a way, the answer to (S5) would be:

(T6) : $P_{1}$ is better than $P_{2}$ because people have $I_{1} \ldots I_{n}$, and $P_{1}$, having $E_{1} \ldots E_{n}$, fulfills those interests, while $P_{2}$, lacking $E_{1} \ldots E_{n}$, does not.

(T6) presuposses (S4) has been refuted. For the skeptic, it is a matter of taste whether a poem has consistence, unity, originality, and so on. The contemporary version of skepticism, as represented by Frank Sibley, asserts that esthetical terms, such as 'unity' and 'consistence', lack such qualities as would sufficiently determine their right use. Assuming, however, that people have certain needs and interests which poetry fulfills, those same needs provide an empirical basis for evaluation.

For Milton H. Snoyenbos, then, the evaluation of poetry is a functional kind of evaluation. If we concede there are empirically determinable interests and needs which poetry fulfills, we shall be in a position to investigate, in an empirical way, which qualities give a poem a high level of ability to fulfill those interests and needs. In short, the functional approach, plus a careful explanation of interests and needs, may well provide a basis to understand the admittedly rich complexity of poetic evaluation.

(Summary by Sebastián Lamoyi) 